Proceedings of the 1999 Particle Accelerator Conference, New York, 1999

\title{
ELECTRON COOLING ASSISTED BEAM ACCUMULATION IN THE HEAVY ION SYNCHROTRON SIS BY REPEATED MULTITURN INJECTION
}

\author{
M. Steck, L. Groening, K. Blasche, H. Eickhoff, B. Franczak, B. Franzke, T. Winkler, \\ GSI Darmstadt, Germany; V.V. Parkhomchuk, BINP Novosibirsk, Russia
}

\begin{abstract}
The heavy ion synchrotron SIS has been equipped with an electron cooling system for fast beam accumulation at the injection energy. Optimization of injection and cooling resulted in synchrotron pulses with more than an order of magnitude higher intensity. For highly charged ions the intensity is limited by recombination with free electrons. The rate coefficients have been measured thus allowing a selection of the most favorable charge state in order to maximize the beam intensity for highly charged ions.
\end{abstract}

\section{INTRODUCTION}

The heavy ion synchrotron SIS [1] is filled by horizontal multiturn injection which results in a horizontal emittance $\epsilon_{x} \simeq 150 \pi \mathrm{mm}$ mrad. It can presently not be operated with highly charged ions up to the space charge limit. The intensity for beams of highly charged heavy ions is limited by the ion source and the low energy section of the injector linac. In order to facilitate beam accumulation by repeated multiturn injection with interspersed cooling an electron cooling system has been designed [2].

Short transverse cooling times allow fast repetition of multiturn injection into the outer part of the horizontal ring acceptance, while the inner part is reserved for the cooled accumulated ion beam. As the typical synchrotron cycle times are a few seconds and transverse cooling times of order $100 \mathrm{~ms}$ are aimed at the intensity gain can be up to one order of magnitude. The cooling time decreases proportional to $A / q^{2}$ promising the highest intensity gain for the heaviest ions which can be injected with the lowest intensities.

After an intensity upgrade of the linac the electron cooler will be ready for accumulation of beams of rare isotopes and preparation of high quality beams.

\section{ELECTRON COOLING SYSTEM}

The electron cooling system was designed and manufactured in a collaboration between GSI and BINP, Novosibirsk. The design parameters of the electron cooling system [2] were specified according to the requirement of transverse cooling times for ions with mass numbers $A \geq 100$ of around $100 \mathrm{~ms}$. Use of adiabatic magnetic expansion for reduction of the transverse electron temperature and for matching of the electron beam diameter to the ion beam size was included in the design. Cooling at an

$\begin{array}{lr}\text { electron energy } & 6.3 \mathrm{keV} \\ \text { cathode diameter } & 25.4 \mathrm{~mm} \\ \text { cathode temperature } & \sim 1200 \mathrm{~K} \\ \text { gun perveance } & 2.9 \mu \mathrm{P} \\ \text { maximum electron current } & 1.5 \mathrm{~A} \\ \text { relative loss current } & \leq 1 \times 10^{-4} \\ \text { magn. expansion factor } & 3 \\ \text { magn. field strength in cool. section } & 0.06 \mathrm{~T} \\ \text { magn. field parallelism in cool. section } \quad \leq 1 \times 10^{-4} \\ \text { vacuum pressure } & \leq 1 \times 10^{-10} \mathrm{mbar}\end{array}$

Table 1: Typical parameters of the SIS electron cooling device for ion beam accumulation.

intermediate energy (between 55 and $65 \mathrm{MeV} / \mathrm{u}$ ) will allow rebunching at the second harmonic of the revolution frequency for generation of high density ion bunches.

The complete electron cooling system was assembled at GSI and tested with electron beam outside the synchrotron before installation in the ring tunnel [3]. The typical parameters of the electron cooling device for operation at the injection energy of the synchrotron, which requires an electron energy of $6.3 \mathrm{keV}$, are listed in Table 1.

The control hardware for the electron cooling system has been prepared for ramped operation synchronized with the acceleration cycle. Since the cooler has been used for beam accumulation at the injection energy to date operation with static magnetic field and constant electron energy and current was sufficient for commissioning in the synchrotron.

\section{SYNCHROTRON OPERATION WITH ELECTRON COOLER}

For the operation of the electron cooler in one straight section of the synchrotron additional corrections of the closed orbit had to be implemented. A small dipole magnet at both ends of the cooler and the correction coils in the adjacent main dipole magnets correct the horizontal kick introduced by the toroids and allow an adjustment of the ion beam displacement and the beam direction in the cooling section. The coupling of horizontal and vertical phase space due to the longitudinal field of the cooler is not corrected. For optimum injection the ring tune and the setting of the injection beam line have to be fine tuned after powering of the cooler magnetic field and of the closed orbit corrections. Multiturn injection with the cooler magnetic fields presently results in about $65 \%$ of the standard multiturn gain factor. 
For beam accumulation with cooling the amplitude of the orbit bump in the injection section is slightly reduced. Thus free space between the closed orbit and the electrostatic septum is provided for the cooled circulating beam to pass by the septum when new beam is injected. Beam accumulation starts when the electron beam is switched on with the electron velocity matched to the ion velocity after injection. Additional fine adjustment of the spatial and angular alignment between electron and ion beam minimizes the transverse cooling time as well as the emittance of the cooled beam. The intensity of the ion beam can be increased by more than one order of magnitude after proper setting of all cooler and ring parameters (Fig. 1).

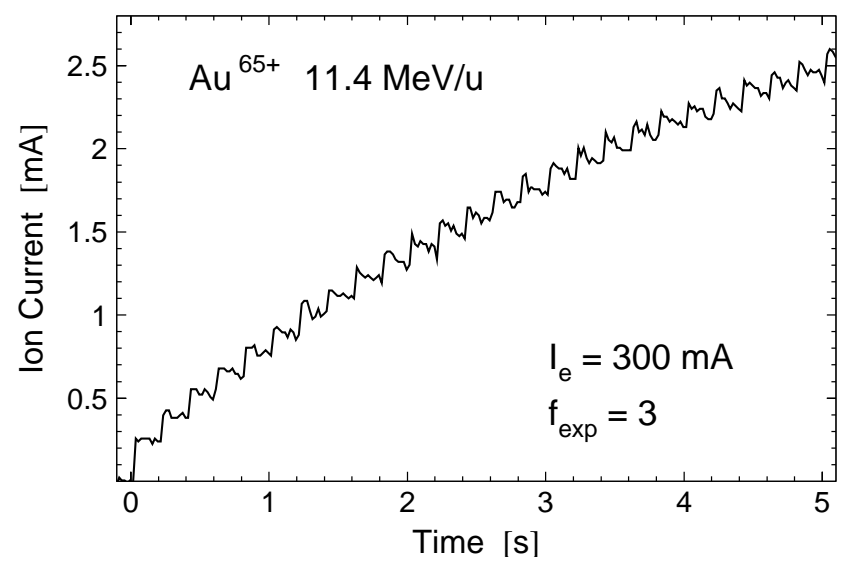

Figure 1: Accumulation of $1 \times 10^{9} \mathrm{Au}^{65+}$ ions in less than $5 \mathrm{~s}$. The ion beam was cooled at the injection energy with a $300 \mathrm{~mA}$ electron beam expanded by a factor of 3 .

\section{BEAM ACCUMULATION}

For high average beam intensity from the synchrotron the accumulation rate, i.e. the intensity filled into the synchrotron per unit of time divided by the current in the injection beam line, has to be maximized. Systematic variations of the injection and cooler parameters were performed to find the optimum accumulation conditions. Figure 2 shows the influence of the electron beam current for three different electron beam expansion factors on the accumulation rate for optimized injection rate. An expansion factor of three and typical electron currents of $0.3-0.5 \mathrm{~A}$ are usually applied for beam accumulation. Higher electron currents result in slightly faster accumulation, but the recombination losses also increase and limit the maximum intensity (see Sect. 5).

The variation of the accumulation rate with the time between successive injections exhibits a maximum for rather short time intervals (Fig. 3). A time of about $200 \mathrm{~ms}$ is required for transverse cooling, for larger time intervals the additional cooling time will not accumulate the beam more efficiently and the accumulation rate decreases proportional to the injection rate.

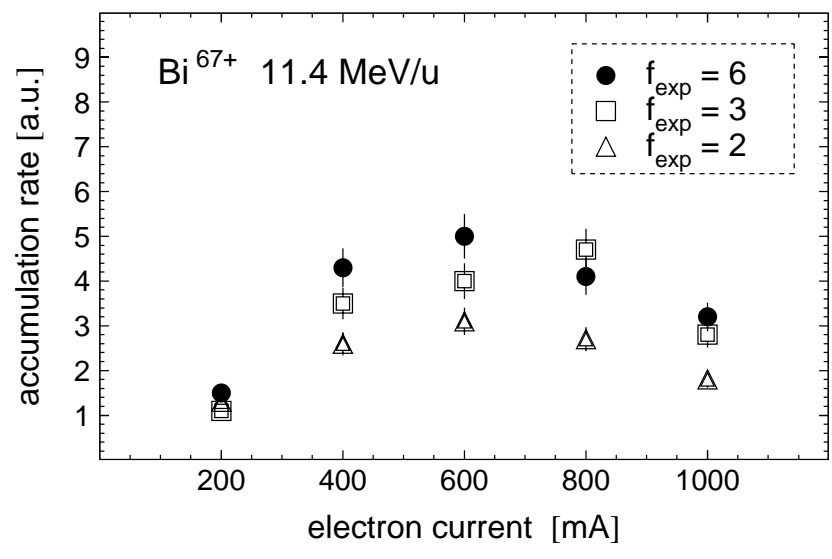

Figure 2: Accumulation rate as a function of the electron current for different expansion factors.

The maximum intensity of the ion beam usually saturates on a certain intensity level after some ten injections. Two diverse processes which limit the beam intensity have been observed. For light ions which can be injected with higher intensity from the injector (more than $10^{8}$ ions after a single multiturn injection) beam instabilities arise due to the high phase space density of the ion beam. A maximum intensity of $7 \times 10^{9} \mathrm{Kr}^{34+}(7 \mathrm{~mA})$ has been achieved [4]. The ion beam approaches the space charge limit, but also coherent transverse oscillations may be excited by interaction with transverse impedances. A transverse feedback system is presently designed for damping of coherent oscillations.

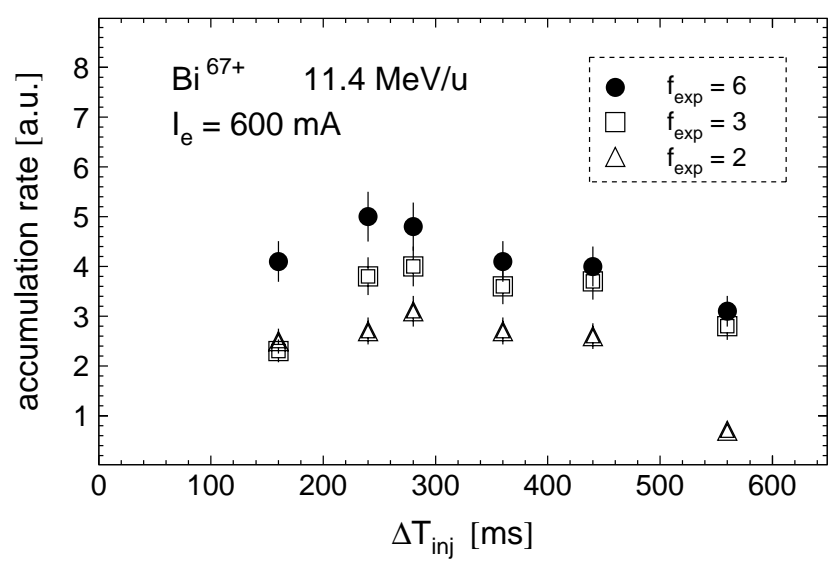

Figure 3: Accumulation rate versus the time interval between successive injections.

With highly charged heavy ions the limitation of the maximum beam intensity unambiguously is related to beam losses by recombination with free electrons in the cooling section which have been studied in more detail. 


\section{RECOMBINATION OF HIGHLY CHARGED IONS}

Before injection into the synchrotron the ions are usually stripped at the injection energy $11.4 \mathrm{MeV} / \mathrm{u}$ in order to reach the highest final energy. At the injection energy heavy ions are produced with $10-20$ bound electrons in largest abundance.

The recombination rate for two species of highly charged ions was studied experimentally for the various charge states available after the injection line stripper. The recombination rate was evaluated from the exponential decrease of the ion current when injection was stopped and the ion beam continued to circulate at the injection energy. Measurements of the beam lifetime for different electron currents allowed to distinguish between losses due to recombination with electrons in the cooler and the losses caused by interaction with the residual gas. The recombination rate with electrons showed a linear increase with electron density and for fixed electron current a scaling inversely proportional to the square root of the expansion factor as expected [5].

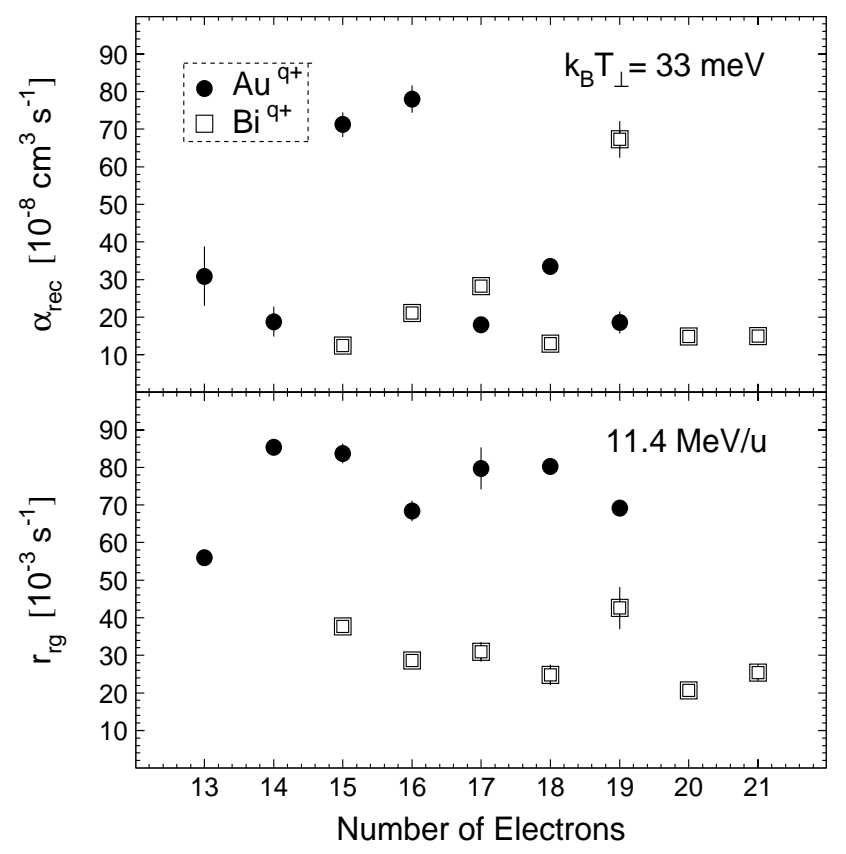

Figure 4: Rate coefficient $\alpha_{r e c}$ for recombination with free electrons and recombination rate in the residual gas $r_{\mathrm{rg}}$ measured for the electron configurations of $\mathrm{Au}$ and $\mathrm{Bi}$ ions available at the synchrotron injection energy $11.4 \mathrm{MeV} / \mathrm{u}$.

The results of measurements of the rate coefficient for recombination with free electrons and the recombination rate in the residual gas for $\mathrm{Au}$ and $\mathrm{Bi}$ charge states with the various numbers of bound electrons that are available after the stripper in the SIS injection line is shown in Fig. 4. The loss rate in the residual gas can be reasonably explained by recombination with bound electrons of the gas constituents which is the dominant charge changing process at SIS en- ergies. The difference between $\mathrm{Au}$ and $\mathrm{Bi}$ is likely to be caused by changes in the vacuum pressure and the composition of the residual gas between the two experiments.

The recombination rate in the electron beam is in disagreement with calculations of radiative electron capture which is expected to be the dominant recombination process. Neither the absolute value of the rate coefficient nor the variations with the charge state which can amount up to a factor of five are comprehensible assuming radiative electron capture only. The recombination rate maxima for the two ions are not correlated with the electronic configuration. Resonant electron capture at small relative energy, e.g. by dielectronic recombination, could be an explanation for the enhanced recombination rates and the strong variations with the charge state which have been reported previously [6].

The charge states with high recombination losses must be avoided if accumulation of the maximum beam intensity is aimed at. Even for the more favorable charge states the beam lifetime due to recombination losses with free electrons is below $10 \mathrm{~s}$ for the typical electron densities of $3-5 \times 10^{7} \mathrm{~cm}^{-3}$, the lifetime due to the residual gas is on the order of some $10 \mathrm{~s}$ depending on the vacuum conditions. Therefore for the heaviest ions a careful choice of the most favorable charge state with respect to recombination is mandatory to take greatest advantage of beam accumulation. After selection of the most favorable charge state $\mathrm{Au}^{65+}$ the number of ions in a SIS pulse could be increased to $1.5 \times 10^{9}$ which is a factor of 15 higher than the maximum intensity achieved ever for gold beams.

\section{REFERENCES}

[1] K. Blasche, B. Franzke, Proceedings of the $4^{\text {th }}$ European Particle Accelerator Conference, London, 1994, edited by V. Suller and Ch. Petit-Jean-Genaz (World Scientific, Singapore, 1994) 133.

[2] M. Steck, K. Blasche, W. Bourgeois, B. Franzke, L. Groening, N.S. Dikansky, V.I. Kudelainen, V.V. Parkhomchuk, A.I. Sharapa, A.V. Shemyakin, B.M. Smirnov, Proceedings of the $5^{\text {th }}$ European Particle Accelerator Conference, Sitges, 1996, (World Scientific, Singapore, 1996) 1185.

[3] L. Groening, M. Steck, T. Winkler, V.I. Kudelainen, V.V. Parkhomchuk, A.I. Sharapa, A.V. Shemyakin, B.M. Smirnov, Proceedings of the $6^{\text {th }}$ European Particle Accelerator Conference, Stockholm, 1998, (Institute of Physics Publishing, 1998) 1034.

[4] K. Blasche, H. Eickhoff, B. Franczak, B. Franzke, L. Groening, M. Steck, T. Winkler, V.I. Kudelainen, V.A. Dolgashev, V.V. Parkhomchuk, Proceedings of the $6^{\text {th }}$ European Particle Accelerator Conference, Stockholm, 1998, (Institute of Physics Publishing, 1998) 550.

[5] L. Groening, PhD Thesis, GSI DISS. 98-20.

[6] J. Bosser, J. Broere, C. Carli, M. Chanel, C. Hill, R. Maccaferri, S. Maury, D. Möhl, G. Molinari, S. Rossi, E. Tanke, G. Tranquille, M. Vretenar, Proceedings of the $6^{\text {th }}$ European Particle Accelerator Conference, Stockholm, 1998, (Institute of Physics Publishing, 1998) 253. 